\title{
Der Verein im Blickfeld der Gerichte
}

\author{
VON YVONNE JORDAN UND \\ FRIEDRICH VOGELBUSCH \\ Yvonne Jordan ist Mitarbeiterin, \\ Prof. Dr. Friedrich Vogelbusch ist \\ Partner der Wirtschaftsprüfungs- \\ gesellschaft Warth \& Klein Grant \\ Thornton AG. \\ www.wkgt.com
}

\author{
Viele eingetragene Verein sind "unternehmerisch" \\ tätig, beispielsweise weil sie eigene Einrichtungen \\ wie Beratungsstellen oder Kindergärten betreiben. \\ Dieses "Nebenzweckprivileg" für den Idealverein \\ steht seit einiger Zeit in der Diskussion.
}

In den letzten Jahren wurde aufgrund von Gerichtsurteilen die rechtliche Basis vieler diakonischer, karitativer und kirchlicher Betätigungen grundsätzlich in Frage gestellt. Im Normalfall haben sich gemeinnützige Vereine weniger für die Rechtsprechung zu vereinsrechtlichen Fragen interessiert als für die Rechtsprechung der Steuergerichte (Finanzgerichte bzw. Bundesfinanzhof). Dass nun die Rechtsprechung der Zivilgerichte von hoher Brisanz ist, hängt mit der bisherigen großzügigen Rechtsprechung und dem entsprechenden großzügigen Verwaltungshandeln der Vereinsregister zusammen. De facto wurde ein Abweichen der praktischen Handhabung vom Gesetzeswortlaut erlaubt.

Das Kammergericht (KG) Berlin hatte mit Urteil vom 18. Januar 2011 (1) die Rechtsform des eingetragenen Vereins (e. V.) als nicht geeignet für den Betrieb eines gemeinnützigen Zweckbetriebs (z. B. Kindergarten, Jugendbetreuung, Pflegeheim, Werkstatt für behinderte Menschen) eingeordnet. Im jüngsten Fall des KG Berlin vom 7. März 2012 (Az. 25 W 95/11) wurde diese Rechtsauffassung noch einmal bestätigt. Das bisher geltende Nebenzweckprivileg für eingetragene Vereine wurde vom Berliner Gericht aufgegeben. Andere Urteile, die zu dieser Frage zwischenzeitlich ergangen sind, sehen die Lage etwas differenzierter (2).

In einigen Bundesländern haben Vereinsregister im vorigen Jahr begonnen, die Kultur- und Sozialbetriebe in der Rechtsform des eingetragenen Vereins anzuschreiben und eine Umwandlung in eine andere Rechtsform (z. B. die Genos- senschaft) bzw. die Ausgliederung des Zweckbetriebes in eine Tochter-GmbH (Ausgliederung) zu verlangen.

In diesem Beitrag sollen die rechtlichen Grundlagen dieser Diskussion dargestellt und besprochen werden. Zudem sind die rechtlichen Konsequenzen für die Vereine im karitativen und kirchlichen Bereich zu ermitteln.

\section{Das Nebenzweckprivileg für eingetragene Vereine}

Das Problem der wirtschaftlichen Betätigung ergibt sich aufgrund einer engen Legaldefinition des Idealvereins $(\mathbb{S} 21$ BGB). Der Idealverein ist die Vereinsform, welche in Vereinsregister eingetragen werden kann. Der nicht eintragungsfähige Verein ist der sogenannte wirtschaftliche Verein, der in $\$ 22$ BGB definiert wird. Er erlangt seine Rechtsfähigkeit durch Konzession (3).

Der eingetragene Verein ist von seiner rechtlichen Konstruktion her auf die Verfolgung eines ideellen Zwecks gerichtet. Im Zivilrecht ist folgendes zum nicht wirtschaftlichen Verein vorgegeben: »Ein Verein, dessen Zweck nicht auf einen wirtschaftlichen Geschäftsbetrieb gerichtet ist, erlangt Rechtsfähigkeit durch Eintragung in das Vereinsregister des zuständigen Amtsgerichts. « $(\mathbb{} 21$ BGB Nicht wirtschaftlicher Verein)

Es ist seit vielen Jahren gängige Praxis, dass es einem eingetragenen Verein unter dem sogenannten Nebenzweckprivileg gestattet wurde, sich wirtschaftlich zu betätigen. Diese Betätigung wurde als unschädlich für den Idealverein 
angesehen, sofern sie im Nebenzweck stattfindet und das Handeln des Vereins nicht überwiegend prägt. Die tatsächliche Tätigkeit gibt den Ausschlag für die Einordnung des Vereinsvorhabens. Die deklarierten Satzungsziele sind dagegen nur von untergeordneter Bedeutung (4).

Dieser Rechtsprechung folgend hat sich die Praxis der Registergerichte bezüglich der Eintragung von Vereinen, die Träger sind für einen Geschäftsbetrieb (5), vom eng gefassten Gesetzestext gelöst. Traditionell sind selbst solche Vereine als Idealvereine anerkannt, die Millionenumsätze erwirtschaften und ein großes Vermögen aufweisen.

Neben dem Idealverein sieht das Zivilrecht den wirtschaftlichen Verein vor: »Ein Verein, dessen Zweck auf einen wirtschaftlichen Geschäftsbetrieb gerichtet ist, erlangt in Ermangelung besonderer bundesgesetzlicher Vorschriften Rechtsfähigkeit durch staatliche Verleihung. Die Verleihung steht dem Land zu, in dessen Gebiet der Verein seinen Sitz hat.«( $(22$ BGB: Wirtschaftlicher Verein)

Der nichtwirtschaftliche Verein erlangt Rechtsfähigkeit durch seine Eintragung in das Vereinsregister, während der wirtschaftliche Verein nur durch staatliche Verleihung rechtsfähig wird. Bei dem nicht eingetragenen Verein haf- ten die für ihn in seinem Namen Handelnden persönlich (6). Die Haftung bei dem in das Vereinsregister eingetragenen Verein ist hingegen so geregelt, dass grundsätzlich nur der Verein als solcher haftet ( $\mathbb{S} 31 \mathrm{BGB})$, nicht aber der Vorstand oder gar die Mitglieder.

Eine Tabelle (vgl. Abbildung) mag einen Überblick über die bisherige traditionelle Rechtsprechung mit Beispielen zur Einordnung von Idealvereinen und wirtschaftlichen Vereinen geben (7).

\section{Die bisherige Auslegung und Bedeutung des Nebenzweckprivilegs}

Bisher wurde in einem zweistufigen Verfahren, das auf eine Grundsatzarbeit von Karsten Schmidt zurückgeht (vgl. Schmidt: Verbandszweck und Rechtsfähigkeit im Vereinsrecht, 1984), geprüft:

1. liegt ein wirtschaftlicher Geschäftsbetrieb vor, der a. am Markt (d. h. nach außen hin) tätig wird, b. nur auf einem Binnenmarkt gegenüber den Vereinsmitgliedern tätig wird oder c. der dem Zweck der genossenschaftlichen Kooperation dient.

2. dient die wirtschaftliche Betätigung dem Vereinszweck und ordnet sie sich diesem funktionell unter.
Die Möglichkeit, trotz wirtschaftlicher Betätigung als Idealverein anerkannt zu werden, wird als »Nebenzweckprivileg« bezeichnet. Nach der diese Prinzip zugrunde liegenden Rechtsprechung (8) hat die Verwaltungspraxis typologisch betrachtet, ob bei einem einzutragenden Verein ein wirtschaftlicher Hauptzweck oder in erster Linie ein ideeller Zweck verfolgt wurde.

Die langjährige Rechtsprechung hatte sich damit befasst, welche Voraussetzungen für das Nebenzweckprivileg gelten.

Die Anwendung des Nebenzweckprivilegs hat sich nicht allein auf quantitative Kriterien wie Umsatzerlöse oder das Vermögen bezogen. Wenn beispielsweise ein Vergleich der Einnahmen oder Aufwendungen aus dem Betrieb mit denjenigen im Rahmen der ideellen Betätigung alleine bedeutsam gewesen wäre, hätte ein großer Verein, der sich mit dem Geschäftsbetrieb Mittel für seine ideelle Betätigung beschaffen wollte, keine Anerkennung als Idealverein erlangen können.

Vielmehr stellte die herrschende Lehre darauf $a b$, ob eine qualitative Zweck-Mittel-Relation gegeben war. Die wirtschaftliche Tätigkeit durfte nicht der einzige verfolgte Zweck sein.
Einordnungen

durch

Gerichtsurteile (Az.)
Berufs- und Interessenverbände, soweit hier die Förderung der allgemeinen beruflichen Interessen und die Beratung und Betreuung der Mitglieder im Vordergrund steht (OLG Stuttgart, Rpfl. 1970, 334),

Arbeitgeberverbände (RGZ 85, 256); Kassenärztliche Vereinigungen (RGZ 83, 231; aber dagegen LG Bonn v. 1.7.1985, MDR 1986, 53); der Verein Creditreform (KG in DNotZ 1928, 247),

Vereine zur Förderung der Interessen von Gewerbetreibenden durch Öffentlichkeitsarbeit (OLG Bremen v. 7.9.1988, Rpfl. 1988, 532)

Verein zur Förderung gewerblicher Interessen (RG v. 8.12.1911, RGZ78, 78 (80)),

Haus- und Grundbesitzerverein (RG v.22.6.1916, RGZ 88, 334; BFH VII R 168/82 V. 4.10.1983, BB 1984, 459),

Verein der Kassenärzte

(RG v. 30.10.1913, RGZ 83, 231),

Lohnsteuerhilfeverein (OLG Celle v. 1.10.1975, NJW 1976, 197);
Verein der Immobilienmakler zum Abgleich von Angebot und Nachfrage (OLG Celle v. 5.5.1995, NJW-RR 1996, 1502; OLG Düsseldorf V. 24.1.1996, NJW-RR 1996, 989),

Verein zur privatärztlichen Verrechnungsstelle (LG Hagen v. 10.3.1959, Rpfl. 1959, 348),

Technische Prüf- und Vertriebsstelle als Verein (LG Oldenburg v. 30.5.1978, Rpfl. 1978, 371);

Abmahnverein (BGH I ZR 52/86 v. 19.5.1988, NJW-RR 1988, 1444 und BGH I ZR 56/89 V. 5.10.1989, NJW-RR 1990, 102; K. Schmidt NJW 1983, 1520),

Verein zur Förderung von Datenkommunikation über das Internet (OFD Münster v. 6.2.1996, BB-1996, 676),

Verein, der seinen Mitgliedern Preisvergleiche und Produktinformationen über das Internet anbietet (LG Saarbrücken v. 24.8.1999, Rpfl. 2000, 25),

Verein zur Koordination als Taxi-Zentrale (BGH II ZB 2/66 v. 20.3.1979, BGHZ 45, 395; OLG Frankfurt v. 19.2.1965, BB 1966, 52),

Time-sharing-Vereine zu Ferienwohnungen

(BayOLG v. 6.4.1989, DNotZ 1990, 103),

in Vereinsform organisierte Scientology-Kirche, die ideelle Güter nach Art von Wirtschaftsgütern vermarktet (VG Stuttgart v. 30.9.1993, NVwZ 1994, 612). 
Solange die wirtschaftlichen Einnahmen dem Vereinszweck gedient haben, war noch eine Anerkennung als nichtwirtschaftlicher Verein nach $\$ 21$ BGB möglich.

Die unternehmerische Tätigkeit musste durch den Vereinszweck gedeckt sein. Bei »natürlicher Betrachtungsweise " musste sich die wirtschaftliche Betätigung als ein die ideelle Betätigung ergänzendes, noch objektiv sinnvolles Mittel zur Förderung des Vereinszwecks darstellen (9).

Weitere Kriterien, wie die Gewinnerzielungsabsicht, die Finanzierung über öffentliche Zuschüsse, die Art und Herkunft der Entgelte oder das Angebot »ehrenhalber « Arbeitsleistungen durch die Mitglieder des Vereins sind nach der Rechtsprechung nicht ausschlaggebend für die Anwendbarkeit des Nebenzweckprivilegs.

\section{Neue Auffassung der Rechtsprechung zur Abgrenzung zwischen Idealverein und wirtschaftlichem Verein}

In den letzten Jahren ist eine Entwicklung in der Rechtsprechung zum Idealverein $\mathrm{zu}$ beobachten, die strengere Kriterien an die Eintragung eines Idealvereins nach $\mathbb{} 21 \mathrm{BGB}$ stellt und das $\mathrm{Ne}$ benzweckprivileg damit immer stärker aushöhlt. Diese Entwicklung gipfelt in dem genannten Urteil des Kammergerichts Berlin. Die neue Auffassung zur Abgrenzung zwischen Idealverein und wirtschaftlichem Verein lautet: Sobald ein Verein einen Geschäftsbetrieb in nennenswertem Umfang unterhält bzw. zu unterhalten beabsichtigt, ist er nicht mehr als e. V. einzutragen.

Die Entscheidung des Kammergerichts Berlin vom 18. Januar 2011 betraf eine Kindertagesstätte. Das Gericht gab dem klagenden eingetragenen Verein vor, sich in eine gemeinnützige Gesellschaft mit beschränkter Haftung, in eine gemeinnützige Unternehmergesellschaft oder gemeinnützige Genossenschaft umzuwandeln.

Das Gericht begründet dies damit, dass Vereinigungen mit wirtschaftlicher Zielsetzung eine handelsrechtliche Rechtsform zu wählen haben, um die Sicherheit des Rechtsverkehrs und insbesondere den Gläubigerschutz zu gewährleisten. Zwar stelle die Bildung und Betreuung von Kindern einen ide- ellen Zweck dar, der dauerhafte Betrieb einer Kindertagesstätte sei jedoch nur mit der Erhebung von Entgelten zur Deckung der sachlichen und personellen Kosten möglich. Von daher sei der Verein gezwungen, sich am Markt unternehmerisch zu betätigen. Wenn diese wirtschaftliche Tätigkeit den Verein hauptsächlich prägt, komme eine Eintragung als Idealverein nicht mehr in Frage. Idealvereine seien durch Ehrenamt gekennzeichnet. Wenn eine vergütete (hauptamtliche) Vorstandsarbeit vorliege und juristische Personen Mitglied in einem Verein sind, lägen Indizien vor, die gegen einen Idealverein sprechen. Die staatliche Finanzierung, die fehlende Gewinnerzielungsabsicht oder die steuerliche Gemeinnützigkeit stellen keine ausreichenden Indizien für einen Idealverein dar.

Im jüngsten Fall, den das KG Berlin am 7. März $2012 \mathrm{zu}$ entscheiden hatte (Az. 25 W 95/11), ist das Gericht davon ausgegangen, dass der Antragsteller die Förderung der Klaviermusik (Unterstützen und Ausbilden von Pianisten und Komponisten) im Wesentlichen nur durch entgeltliche Konzerte und Veröffentlichen von entgeltlicher Klaviermusik erreichen könne, was einen wirtschaftlichen Geschäftsbetrieb von nicht nur untergeordneter Bedeutung erfordere. Dann aber könne er aus vereinsrechtlicher Sicht kein Idealverein sein, auch wenn er nach seiner Satzung steuerbegünstigt ist.

Demgegenüber stehen die Feststellungen des OLG Schleswig-Holstein in seinem Beschluss vom 18. August 2012: Der Betrieb einer Kindertagesstätte dient der Förderung und Betreuung von Kindern. Dies stelle grundsätzlich einen ideellen Zweck dar. Vereine mit der Tätigkeit auf dem Gebiet von Erziehung und Bildung verfolgen in der Regel nichtwirtschaftliche Zwecke, daher sei die automatische Schlussfolgerung, dass Kindertagesstätten Wirtschaftsunternehmen gleichzusetzen wären, unzulässig. Dies gilt nicht zuletzt deswegen, weil der Gesetzgeber in Schleswig-Holstein Vereine als Träger von Kindertagesstätten ausdrücklich vorsieht. Der Gläubigerschutz werde bei Kindertagesstätten durch den Anspruch auf Finanzierung gewährleistet. Der gesetzlich vorgesehene Kostenbeitrag der Eltern könne nicht als Hinweis auf unternehmerische Betätigung gelten. Daher seien Mitgliedsbeiträge nicht als Ent- gelt für eine Dienstleistung, sondern als Mittel zur Erfüllung des ideellen Zwecks zu sehen. Zusätzlich weise die Gemeinnützigkeit auf eine nichtwirtschaftliche Tätigkeit hin.

\section{Kritische Würdigung der Entscheidungen}

Zunächst fällt beim kritischen Vergleich der Argumentationen der beiden Gerichte eine grundlegende Differenz auf, die darauf zurückzuführen ist, dass sich die zu beurteilenden Sachverhalte des KG Berlin und des OLG Schleswig-Holstein hinsichtlich des Umfangs der Betätigung unterscheiden. Während das KG Berlin über eine größere Einrichtung zu befinden hatte, die hauptamtliches Personal beschäftigt, betraf die Entscheidung des OLG Schleswig-Holstein eine kleine Elterninitiative. Die Finanzierung der gegenseitigen Kinderbetreuung durch die Eltern gilt als Kleinsteinrichtung. Eine solche auf Eigeninitiative und gegenseitige Selbsthilfe beruhende Einrichtung wird nach Einschätzung des Gerichts nicht am Markt als Unternehmen tätig. Während kleine Einrichtungen demnach als eintragungsfähig eingestuft werden, gilt dies nicht für größere Einrichtungen, bei denen die gemeinsame Erziehung nicht nur durch die Eltern erbracht wird.

Nichtsdestotrotz sind einige inhaltliche Begründungen beider Gerichte aus Sicht der Verfasser in Frage zu stellen. Das Kammergericht Berlin postuliert, dass Vereine mit wirtschaftlicher Zielsetzung wegen des Gläubigerschutzes auf die üblichen handelsrechtlichen Rechtsformen verwiesen werden sollen. Die außen stehenden Gläubiger seien so besser geschützt. Dem steht entgegen, dass Gesellschaftsformen wie die Unternehmergesellschaft oder Limited ebenfalls nur einen begrenzten Gläubigerschutz gewährleisten. Die Behauptung, Gläubigerschutz werde nur im Handels- bzw. Kapitalgesellschaftsrecht verwirklicht, ist nicht überzeugend.

Vielmehr sollte im Einzelfall geprüft werden, ob in Anbetracht der Satzung, des Mitgliederwillens und der tatsächlichen Tätigkeit des Vereins eine Gefährdung des Gläubigerschutzes zu erwarten sein wird. Ist dies nicht der Fall, besteht u. E. nicht von Vornherein die Veranlassung zum Wechsel der Rechtsform. 
Darüber hinaus bewertet das Kammergericht Berlin das Vorliegen eines Idealvereins unabhängig von der steuerlichen Anerkennung der Gemeinnützigkeit. Von den steuerrechtlichen Vorschriften könne nicht auf die Zulässigkeit einer zivilrechtlichen Rechtsform geschlossen werden. Gegen eine solche strikte Trennung spricht, dass die Gemeinnützigkeit eine Geschäftsgrundlage der Tätigkeit eines Idealvereins ist. In seiner Satzung verpflichtet sich der eingetragene Verein, die Grundsätze der Gemeinnützigkeit einzuhalten. Dies ist daher konstitutiv für die Vereinstätigkeit. Gemeinnützigkeitsrecht und Zivilrecht lassen sich nicht völlig trennen.

Das Vorliegen der Gemeinnützigkeit im steuerrechtlichen Sinne sollte daher u. E. als Indiz dafür angenommen werden, dass die Voraussetzungen für den Status als Idealverein erfüllt sind. Dies ergibt sich aus den Bedingungen, welche die Finanzverwaltung ansetzt, um die Gemeinnützigkeit von Körperschaften anzuerkennen. Bereits nach den gemeinnützigkeitsrechtlichen Regelungen darf der wirtschaftliche Geschäftsbetrieb in der Gesamtschau nicht zum Zweck an sich werden.

Ein Geschäftsbetrieb hat nach der Finanzverwaltungsrichtlinie »um des steuerbegünstigten Zwecks willen « zu erfolgen (vgl. AEAO Ziffer $1 \mathrm{zu} \mathbb{S} 56$ ). Diese Vorgabe orientiert sich am traditionellen Konzept des Nebenzweckprivilegs. Denn auch hier ist ausschlaggebend, dass die unternehmerische Tätigkeit dem Hauptzweck zu- bzw. nachgeordnet ist. Hinzuweisen ist allerdings darauf, dass vor einigen Jahren die bis dahin geltende Geprägetheorie für die Dominanz eines gemeinnützigen Vereins durch einen wirtschaftlichen Geschäftsbetrieb aufgegeben wurde (10).

Die steuerrechtliche Beurteilung sollte nach Ansicht der Verfasser nicht ausgeblendet werden, wenn es um die vereinsrechtlichen Verfahren zur Anerkennung eines Idealvereins geht, der einen Eintrag ins Vereinsregister begehrt.

Allerdings ist auch das Urteil des OLG Schleswig-Holstein in seiner Argumentation nicht vollständig überzeugend. Zum einen stützt es sich auf die mittlerweile als überholt geltende »subjektive Theorie", nach der bei der Bewertung als Idealverein ausschließlich der Zweck des Vereins ausschlaggebend ist. Es kommt aber nach der h. L. nicht nur auf den in der Satzung verankerten Endzweck an, sondern auch auf die tatsächliche Entfaltung unternehmerischer Tätigkeiten. Ein Kindertagesstättenverein, in dem die Eltern als "Kunden « betrachtet werden können, ist durchaus als Verein mit unternehmerischer Tätigkeit in einem Binnenmarkt (s. o.) einzuordnen. Es sollte vielmehr darauf abgestellt werden, ob sich diese wirtschaftliche Tätigkeit dem Zweck des Vereins unterordnet.

Darüber hinaus sollte u. E. zwar die steuerrechtliche Behandlung zumindest als Indiz bei der vereinsrechtlichen Beurteilung Beachtung finden - von dem Status der Gemeinnützigkeit kann jedoch nicht unmittelbar auf das Fehlen eines wirtschaftlichen Geschäftsbetriebes im Sinne des Vereinsrechts geschlossen werden. Der umgekehrte Fall trifft zu: Ist in steuerlicher Hinsicht von einem wirtschaftlichen Geschäftsbetrieb nach $\mathbb{S} 14 \mathrm{AO}$ auszugehen, wobei auch die Zweckbetriebe mit betrachtet werden, ist ein wirtschaftlicher Geschäftsbetrieb im vereinsrechtlichen Sinne anzunehmen. Greift nicht das Nebenzweckprivileg, kommt eine Eintragung als Idealverein nicht in Frage. Im Fall des OLG Schleswig-Holstein konnte jedoch ohnehin nicht auf das Privileg des Nebenzwecks abgestellt werden, da der betreffende Verein ausschließlich die Kindertagesstätte betreibt - und insofern nur ein einziger, und nicht mehrere Zwecke vorliegen.

\section{Weitere Urteile}

Die Abgrenzung von Idealverein und wirtschaftlichem Verein hatte bereits in der Vergangenheit zahlreiche Gerichte beschäftigt. Die vorliegenden Einzelfallentscheidungen geben jedoch keine Klarheit hinsichtlich der aktuell zugrunde zu legenden Kriterien.

Etwa entschied das OLG München in seinem Beschluss vom 28. Mai 2013 (11), dass die Unterstützung bei der Altersversorgung von Betriebsangehörigen und deren Familien durch eine eigens hierfür eingerichtete Kasse nicht als wirtschaftliche Tätigkeit einzuordnen ist.

In einem anderen Fall verfolgte ein Heimat-Verein den Zweck der »finanziellen und ideellen Unterstützung von örtlichen, dem Gemeinwohl dienlichen
Einrichtungen«. Hierfür wurden Grundstücke erworben, um sie den Betreibern von Windkraftanlagen gegen Entgelt zu überlassen. Das OLG Thüringen entschied in seinem Beschluss vom 30. Oktober 2012 (12), dass eine Eintragung ins Vereinsregister nicht vorgenommen werden darf. Zur Begründung hieß es, dass durch die Vermarktung der Immobilien der größte Teil der Vereinsmittel erwirtschaftet werden. Die Vermarktung sei als wirtschaftliche Tätigkeit einzustufen und werde nicht mehr durch das Nebenzweckprivileg abgedeckt. Die Verwendung der Erlöse für ideelle Zwecke sei nicht in die Entscheidung einzubeziehen.

Es bleibt also festzuhalten, dass eine moderate wirtschaftliche Tätigkeit für den Status als Idealverein und somit für die Eintragung ins Vereinsregister nicht schädlich sein muss, sofern sie der eigentlichen Zweckverfolgung dient. Insbesondere Betätigungen, die mit einer einfachen Vermögensverwaltung einhergehen und wenig personal- und zeitaufwendig sind, dürften grundsätzlich in den Bereich des Nebenzweckprivilegs fallen.

Diese Art der Argumentation zeigt auf, dass es den bisherigen Entscheidungen an Eindeutigkeit der zugrunde gelegten Kriterien mangelt. Nur die Tatsache, dass der größte Anteil der Vereinsmittel durch wirtschaftliche Tätigkeiten generiert wird, kann nicht für die Feststellung ausreichen, dass die wirtschaftliche Tätigkeit faktisch zum Vereinszweck selbst wird. Der Betrieb von Unterstützungskassen kann im Gegensatz zu der langfristigen Verwaltung von Immobilien durchaus erhebliche Zeit- und Personalressourcen beanspruchen. In beiden beschriebenen Beschlüssen wären daher u. U. auch andere Entscheidungen denkbar gewesen.

\section{Fortgang der Rechtsprechung des Kammergerichtes Berlin}

Das Kammergericht Berlin bestätigt unterdessen seine Rechtsprechung (z. B. im Beschluss vom 7.3.2012): Die satzungsmäßige Formulierung eines Zweckes, der als wirtschaftliche Betätigung aufzufassen ist, soll eintragungsschädlich sein. Ob das Nebenzweckprivileg eine Eintragung dennoch ermögliche, hänge von den jeweiligen Umständen des Einzelfalls ab. Wenn diese im Zuge der 
vereinsregistergerichtlichen Prüfung der Eintragung nicht hinreichend sicher prognostizierbar sind, erfolge die Eintragung im Zweifel nicht.

Die Folge dieser neuen Rechtsprechung ist, dass auch andere soziale und kulturelle Branchen von der engeren Interpretation des Nebenzweckprivilegs betroffen sind. Zum Beispiel könnten auch der Betrieb von Altenheimen für den Zweck "Altenhilfe" oder die Ausrichtung von Veranstaltungen für den Zweck »Kulturförderung « künftig nicht mehr den Anforderungen eines Idealvereins genügen.

Während das Kammergericht Berlin auf die Satzung und tatsächliche Betätigung des Vereins abstellt, berücksichtigt das VGH Baden-Württemberg maßgeblich den Willen und die Überzeugungen der Mitglieder (13). Sind die Leistungen des Vereins untrennbar mit den Überzeugungen des Vereins verbunden und verfügen sie insofern über einen unverwechselbaren Charakter, ist eine vergleichbare Erbringung der Leistung durch andere Marktanbieter nicht möglich. Eine äußere Tätigkeit dient dann der Erhaltung der Existenz- und Funktionsfähigkeit des Vereins und ist nicht als wirtschaftliche Tätigkeit anzusehen. Wirtschaftliche Aktivitäten sind dem-

\section{Konsequenzen der neuen Rechtsprechung}

Wenn Vereinsmitglieder ein Gründungsvorhaben beabsichtigen bzw. wenn bestehende Vereine von ihren zuständigen Registergerichten mit der Aufforderung, einen Rechtsformwechsel vorzunehmen bzw. für die wirtschaftliche Betätigung eine Tochtergesellschaft zu errichten, angeschrieben werden, sollte der anstehende Sachverhalt genau geprüft werden.

In den Fällen, in denen die ideelle Betätigung im Vordergrund steht und die Vereinsverfassung von grundlegender Bedeutung für die Betätigung ist, bestehen nach den dargestellten Grundsätzen gute Aussichten, sich beim Gericht erfolgreich für den Idealverein verwenden zu können. Der eingetragene Verein ist insbesondere dann eine geeignete Rechtsform, wenn das persönliche Engagement der Mitglieder im Vordergrund steht. Dies ist etwa bei Fördervereinen von Kindergärten, Schulen oder bei Orts- und Landesverbänden der Freien Wohlfahrtspflege der Fall, die in erster Linie der Unterstützung der sozialen Arbeit im Allgemeinen dienen und nicht auf den Betrieb eines Sozialbetriebs gerichtet sind.

\section{"Gemeinnützigkeitsrecht und Zivilrecht lassen sich nicht völlig trennen"}

nach nicht schädlich für die Eintragung ins Vereinsregister, falls das Vereinsleben, eher durch Mitgliederinteressen und nicht durch wirtschaftliche Interessen bestimmt wird.

Wenn man die neueren Urteil der Gerichte zum Idealverein insgesamt würdigt, sollte festgehalten werden, dass die engere Auslegung des Wortlauts des \ 21 BGB nicht dazu führen darf, dass keinerlei wirtschaftliche Betätigungen von Idealvereinen mehr möglich bleibt. Damit würde »das Kind mit dem Bade ausgeschüttet «. Vielmehr sollte eine Prüfung in jedem Einzelfall erfolgen.

Die engere Gesetzesauslegung bringt jedoch in jedem Fall mit sich, dass sich viele Vereine mit den zuständigen Registergerichten auseinandersetzen werden sowohl im Falle der Neugründung, als auch bei Umstrukturierungen und Satzungsänderungen.
In den Fällen, in denen alternativ der Rechtsformwechsel in eine gGmbH oder eine Stiftungsgründung möglich erscheint, sollten diese Rechtsformen genutzt werden (14). In einigen Fällen ist es sicherlich sachgerecht, eine zweistöckige Struktur zu schaffen, also dass der eingetragene Verein eine Tochtergesellschaft ausgliedert.

$\mathrm{Zu}$ beachten ist, dass bei allen Umstrukturierungen die Vorschriften des Umwandlungsrechts genutzt werden können. Nach dem Umwandlungsrecht ist es möglich, Betriebe so wie sie stehen und liegen im Wege der Gesamtrechtsnachfolge auf einen neuen Rechtsträger zu übertragen. Bedeutsam ist die Gesamtrechtsnachfolge deshalb, weil alle Rechtsbeziehungen (d. h. Verträge mit Heimbewohnern, Betreuungsverträge mit Kindern oder Jugendlichen, alle Beschäftigungsverhältnisse, Dauerschuld- verhältnisse wie Mietverträge und Darlehen usw.) durch einen Notarvertrag auf den neuen Rechtsträger übertragen werden können.

Der Formwechsel ist eine besondere Form der Umwandlung nach dem Umwandlungsgesetz: Es wird sozusagen nur das Rechtskleid gewechselt, ohne dass Vermögen übertragen wird. Vielmehr besteht die rechtliche und wirtschaftliche Identität des Rechtsträgers fort. Es ändert sich lediglich seine Rechtsform und die rechtliche Struktur ( 190 UmwG). Für den Formwechsel eingetragener Vereine gelten folgende Grundsätze ( $\int 272$ Abs. 1 UmwG): ein Formwechsel vom e.V. aus ist auf folgende neue Rechtsformen möglich:

Kapitalgesellschaften (GmbH, AG) - eingetragene Genossenschaft (15).

Steuerrechtlich ist $\mathrm{zu}$ beachten, dass weitere Tatbestandsmerkmale nach dem Umwandlungssteuergesetz hinzu kommen, beispielsweise ist notwendig, dass bei einer Ausgliederung ein steuerlich anerkannter Teilbetrieb ausgegliedert wird. Der Formwechsel vom Verein zur Kapitalgesellschaft oder Genossenschaft ist ohne Nachversteuerung nur möglich, wenn die Zielrechtsform gemeinnützig ist. Zwischen den Alternativen sollte sorgfältig abgewogen werden, da es um eine grundsätzliche Entscheidung mit langfristiger Wirkung handelt.

Für kirchliche, karitative und diakonische Vereine kann es unter Umständen in Frage kommen, neben dem eingetragenen Verein eine Stiftung zu errichten. Eine Stiftung ist durch den "Ewigkeitscharakter" geprägt, der sich daraus ergibt, dass ein Stifter mit der Übertragung von Vermögen auf eine Stiftung ohne Rückkehrmöglichkeit auf Vermögen verzichtet. Die Stiftung ist eine verselbständigte Vermögensmasse mit eigenen Organen (geschäftsführender Vorstand und Aufsichtsgremium).

Die Stiftung kann ein geeignetes Instrument zur Absicherung eines Geschäftsbetriebes darstellen, da anders als beim eingetragenen Verein keine Abhängigkeit von einer demokratisch bestimmten Mitgliederversammlung besteht. Für die Einwerbung von Spenden und insbesondere Vermächtnissen ist die Stiftung darüber hinaus ein geeignetes Fundraising-Instrument, da 
dem Spender die Sicherheit einer dauerhaften Zweckerfüllung gewährt werden kann. Allerdings ist die Stiftung mit nicht unerheblichen zusätzlichen Anforderungen verbunden (Grundsatz des Vermögenserhalts, Stiftungsaufsicht usw.), so dass eine sorgfältige Prüfung dieser Alternative zur Rechtsform des eingetragenen Vereins geboten erscheint.

\section{Kein Bestandsschutz für bereits eingetragene Vereine}

Für bereits existierende Vereine besteht kein Bestandsschutz. Das Gesetz über das Verfahren in Familiensachen und in den Angelegenheiten der freiwilligen Gerichtsbarkeit ermächtigt das Vereinsregister zur Löschung von Amts wegen, wenn ein Mangel »einer wesentlichen Voraussetzung « vorliegt ( $\$ 395$ FamFG). In diesem Zusammenhang ist darauf hinzuweisen, dass der Verweis auf andere Vereine keine Abhilfe schafft, da nach wie vor der Grundsatz gilt, dass keine Gleichbehandlung im Unrecht zu erfolgen hat.

Vereine haben sich mit dem Vereinsregister auseinanderzusetzen, wenn Satzungsänderungen anstehen, die vorgenommen werden müssen, um veränderte Anforderungen des Gemeinnützigkeitsrechts zu erfüllen. Diese wird durch das Finanzamt erteilt bzw. überwacht und ist wichtige Voraussetzung für Steuerbefreiungen. Die Vereinseintragung, durch die eine eigenständige juristische Person und Haftungsbeschränkung geschaffen wird, erfolgt dagegen durch das Vereinsregister. Das Finanzamt verlangt eine sehr präzise Benennung der realen Tätigkeit in der Satzung des Vereins, um die Gemeinnützigkeit anerkennen zu können. Werden dementsprechend Satzungsänderungen vorgenommen, wird diese durch das Vereinsregister möglicherweise zum Anlass genommen, eine hauptsächliche wirtschaftliche Betätigung festzustellen und den Eintrag zu löschen.

\section{Verfahrensrechtliche Hinweise für einen betroffenen Verein}

Bevor das zuständige Registergericht die Eintragung in das Vereinsregister ablehnt oder den Status als nichtwirtschaftlicher Verein im Nachhinein entzieht, erhalten betroffene Vereine in der
Regel die Möglichkeit zur Stellungnahme. Diese sollte insbesondere den ideellen Gehalt der Einrichtung und den ehrenamtlichen Anteil in der Organisationsstruktur hervorheben.

Eine Ablehnung der Eintragung in das Vereinsregister ergeht in Form eines Beschlusses ( $\$ 382$ Abs. 3 FamFG). Zuvor muss eine angemessene Frist eingeräumt werden, um Eintragungshindernisse zu beseitigen. Bleibt es bei einer Aberkennung des Status als Idealverein, muss gegen diese Entscheidung mit dem Rechtsmittel der Beschwerde vorgegangen werden $(\mathbb{S} \mathbb{S} 382 \mathrm{Abs} .4$ Satz 2, $58 \mathrm{ff}$. FamFG).

Kommt das Registergericht aufgrund einer Überprüfung von Amts wegen oder auf Antrag zu dem Ergebnis, dass bei einem bereits eingetragenen Verein nicht die Voraussetzungen für einen Idealverein vorliegen, so muss der Verein gemäß $\mathbb{3 9 5}$ Abs. 2 Satz 1 FamFG über die beabsichtige Löschung unterrichtet werden. Das Registergericht muss darlegen, welche Aspekte zu einer Neubewertung führen, d. h. beispielsweise die Begründung der Überschreitung des Nebenzweckprivilegs. Zudem muss die Möglichkeit gegeben werden, innerhalb einer angemessenen Frist Rechtsmittel gegen die beabsichtigte Löschung zu erheben (vgl. $\$ 395$ Abs. 2 Satz 1 FamFG). Die Entscheidung über diesen Widerspruch ergeht wiederum in Form eines Beschlusses, der über das Rechtsmittel der Beschwerde anfechtbar ist (16).

Angesichts der erörterten Kritik am Urteil durch das Kammergericht Berlin sind die Erfolgsaussichten zugunsten der Vereine in den Gerichtsverfahren nicht zu unterschätzen. Während der Verfahren sind bestehende Vereine darüber hinaus in ihrer Bestandskraft geschützt. Sind sie jedoch ohne Erfolg, ist ein Wechsel der Rechtsform unerlässlich. Naheliegend wäre die Gründung einer gemeinnützigen Unternehmergesellschaft (uUG) oder einer gemeinnützigen Gesellschaft mit beschränkter Haftung (gGmbH), deren einziger Gesellschafter der Verein ist. Für Elterninitiativen, Alternativ- und Waldorfschulen stellt häufig die Genossenschaft eine geeignete Alternative dar. Konkrete Handlungsempfehlungen sind jedoch vom Einzelfall abhängig und sollten durch eine Rechtsberatung begleitet werden.

\section{Ergebnis}

Seit der Aufgabe der bis dahin geltenden vorherrschenden Interpretation des $\mathbb{S} 21$ BGB im Januar 2011 können sich Vereine nicht mehr auf das Nebenzweckprivileg berufen. Das für die Betätigung vieler karitativer und kirchlicher Vereine bisher geltende Fundament ist daher in Frage gestellt. Bisher konnten die Verantwortlichen von einem großen Beurteilungsspielraum zugunsten der Idealvereine ausgehen. Eine wirtschaftliche Betätigung war solange durch die langjährige Rechtsprechung und Praxis der Vereinsregister gedeckt, solange sich die wirtschaftliche Betätigung funktional dem ideellen Vereinszweck unterordnete.

So ist in den vergangenen Jahrzehnten eine große Zahl von Idealvereinen entstanden, die neben dem Idealzweck einen Geschäftsbetrieb unterhalten haben, in welchen Satzungszwecke umgesetzt wurden. Es war statthaft, dass diese Zweckbetriebe den Einsatz teilweise erheblicher sachlicher und personeller Mittel (und damit Erträge und Aufwendungen zur Finanzierung) vorsehen durften.

Die Eintragungspraxis der Vereinsregister hat sich damit ein Stück weit von der gesetzlichen Definition des $\$ 21$ BGB gelöst. Anders als es der Wortlaut verlangt, war es in der Vereinspraxis zu beobachten, dass der Vereinszweck auf den Betrieb eines Unternehmens mit Entgelten gerichtet war. Die Entgelte waren erforderlich, um die Personal- und Sachkosten zu decken. Wenn ein Verein Träger eines Unternehmens geworden ist, ist er Arbeitgeber geworden, hat Mitarbeiter längerfristig an sich gebunden und nicht unerhebliche Mittel in das Anlagevermögen (Gebäude und Geschäfts- und Betriebsausstattung) investiert.

Nach dieser traditionellen Gesetzesauslegung haben es die Vereinsregister in der Praxis akzeptiert, dass ein auf die Verfolgung ideeller Ziele gerichteter Verein als Nebenzweck einen Zweckbetrieb unterhält. Es musste lediglich sichergestellt sein, dass dieser Geschäftsbetrieb dazu dient, die Satzungszwecke umzusetzen. Der Verein durfte nicht um des Geschäftsbetriebes willen errichtet worden sein. Der wirtschaftliche Zweck durfte nicht alleine verfolgt werden. Ihm musste ein ideeller Zweck übergeordnet werden. 
Das Kammergericht Berlin hat mit seinem Urteilen aus dem Jahre 2011 und in der Folgezeit eine Abkehr von dem Nebenzweckprivileg vorgenommen. In diesem Beitrag wurden die dabei verwendeten Argumente kritisch in Augenschein genommen. Insbesondere das Argument eines besseren Gläubigerschutzes bei den vorgeschlagenen anderen Rechtsformen (Ltd. oder Unternehmergesellschaft) halten die Verfasser nicht für stichhaltig.

Die bestehenden Vereine werden nach Ansicht der Verfasser nicht umher kommen, sich mit der besprochenen Thematik auseinander zu setzen. In den Fällen, in denen das persönliche Engagement der Mitglieder im Vordergrund steht, sollte an der Rechtsform des eingetragenen Vereins festgehalten werden. Ein nebenher als gemeinnützig anerkannter Zweckbetrieb geführter Bereich sollte nicht dazu führen, dass das Rechtskleid gewechselt werden muss. Gegebenenfalls muss der eigene Rechtsstandpunkt durch Einlegen von Rechtsmitteln verteidigt werden. Aufgrund der differenzierten Rechtslage dürften sich in den genannten Fällen ausreichend Argumente für diesen Rechtsstandpunkt finden.

In den Fällen, in denen sowieso aufgrund des gestiegenen Geschäftsumfangs und der günstigeren Corporate Governance die Ausgründung einer $\mathrm{GmbH}$ beabsichtigt gewesen ist, erfolgt durch die engere Gesetzesauslegung der Vereinsregister ein Anschub zum Handeln.

Wenn aus Gründen der Verstetigung der Betätigung bzw. wegen der günstigeren Einwerbung von Spenden und Vermächtnissen die Gründung einer Stiftung in Erwägung gezogen wurde, gilt entsprechendes. Für nicht wenige diakonische, karitative und kirchliche Vereine dürfte der Zeitpunkt für die Verwirklichung einer solchen Gründung nach der neuen Rechtsprechung des Kammergerichtes gekommen sein.

Nur für die Vereine, die sich auf die traditionelle Auslegung des $\mathbb{} 21$ BGB verlassen haben, und die auch weiterhin neben den ideellen Zwecken auch wirtschaftliche Aktivitäten unter dem Vereinsdach betreiben wollen, kann eine schwierige Zeit vorausgesagt werden. Dies gilt insbesondere in einigen Vereinsregisterbezirken, namentlich in Berlin und Sachsen-Anhalt, in denen die Vereinsregister schon aktiv dazu übergegangen sind, die Vereine mit nennenswerten wirtschaftlichen Aktivitäten anzuschreiben und $\mathrm{zu}$ einem Rechtsformwechsel bzw. der Ausgründung einer $\mathrm{GmbH}$ aufzufordern.

Über den Fortgang der Rechtsentwicklung wird in dieser Zeitschrift weiter berichtet werden.

\section{Anmerkungen}

(1) Urteil des KG Berlin vom 18. Januar 2011, Az. 25 W 14/10.

(2) Etwa das OLG Schleswig-Holstein (Schleswig-Holsteinisches OLG, Beschluss vom 18. September 2012, Az. 2 W 152/11).

(3) Nach $\mathbb{} 82$ EGBGB bestimmen die Bundesländer, die Regelungen und Zuständigkeit für die Anerkennung wirtschaftlicher Vereine. In den meisten Bundesländern ist das Innenministerium bzw. das Regierungspräsidium oder die entsprechende Behörde zuständig.

(4) Vgl. OLG Hamm, Beschluss vom 6.9.2007, Az. 15 W 129/07 und KG Berlin vom 26. Oktober 2004, Az. 1 W 269/04.

(5) Steuerrechtlich handelt es sich bei den größeren Geschäftsbetrieb im Regelfall um einen sogenannten Zweckbetrieb, vgl. \$S 65-68 AO.

(6) Vgl. $\$ 54$ S. 2 BGB.

(7) Zitiert nach Schauhoff, Handbuch der Gemeinnützigkeit, 3. Aufl., München 2010, S. 42 f.

(8) Vgl. BGH-Urteil vom 29. September 1982, Az. I ZR 88/80.

(9) Vgl. Waldner/Schweyer: Der eingetragene Verein, 19. Auflage 2010, Rn. 453.

(10) Vgl. BFH Urteil vom 4. April 2007, IR 76/05, in: BStBl. 2007 II, S. 631. Der AEAO hat im Jahre 2012 die bisherige Richtlinie zu $\mathbb{S} 55$ Abs. 1 Nr. 1 aufgegeben.

(11) OLG München, Beschluss vom 28. Mai 2013, Az. 31 W 136/13.

(12) OLG Thüringen, Beschluss vom 30. Oktober 2012, Az. 9 W 415/12.

(13) Vgl. VGH Baden-Württemberg, Urteil vom 12. Dezember 2003, Az. 1 S 1972/00.

(14) Vgl. auch Stefan Winheller in DStR 31/2012, S. $1562 \mathrm{ff}$.

(15) Vgl. auch Stefan Winheller (2012), DStR 31/2012 S. 1562.

(16) Vgl. \$ 393 Abs. 3 Satz 1 und 2 FamFG i.V.m. \$ 395 Abs. 3 FamG.

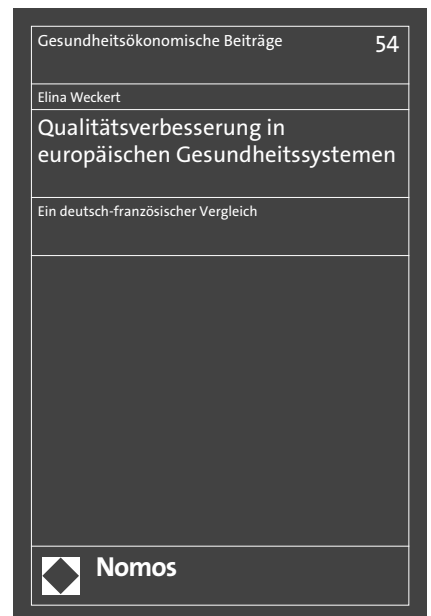

\section{Qualitätsverbesserung} in europäischen

\section{Gesundheitssystemen}

Ein deutsch-französischer Vergleich

Von Dr. Elina Weckert

2014, 399 S., brosch., 88,-€,

ISBN 978-3-8487-1270-0

(Gesundheitsökonomische

Beiträge, Bd. 54)

www.nomos-shop.de/22556

Ein deutsch-französischer Vergleich zeigt, wie die Steuerung der Gesundheitssektoren beider Länder durch das Programm der Qualitätsverbesserung verändert wurde und erklärt Konvergenzen und Divergenzen in der Entwicklung.

Neben der Herausbildung des Programms auf der politischen Agenda werden drei zentrale Instrumente der Qualitätsverbesserung im Detail untersucht: die Qualitätsindikatoren, die medizinischen Behandlungsleitlinien und die Primärarztkonzepte.

Für die Untersuchung wird die Vetospielertheorie mit dem Ansatz der programmatischen Akteure verbunden.

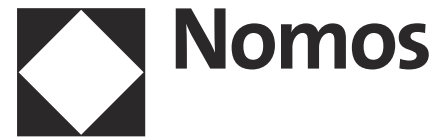

